\title{
Nonlinear dynamics of a regenerative cutting process
}

\author{
Grzegorz Litak • Sven Schubert • Günter Radons
}

Received: 28 April 2010 / Accepted: 23 January 2012 / Published online: 14 February 2012

(C) The Author(s) 2012. This article is published with open access at Springerlink.com

\begin{abstract}
We examine the regenerative cutting process by using a single degree of freedom nonsmooth model with a friction component and a time delay term. Instead of the standard Lyapunov exponent calculations, we propose a statistical 0-1 test analysis for chaos detection. This approach reveals the nature of the cutting process signaling regular or chaotic dynamics. For the investigated deterministic model, we are able to show a transition from chaotic to regular motion with increasing cutting speed. For two values of time delay showing the different response, the results have been confirmed by the means of the spectral density and the multiscaled entropy.
\end{abstract}

Keywords Cutting process $\cdot 0-1$ test $\cdot$ Multiscale entropy

G. Litak $(\varangle)$

Department of Applied Mechanics, Technical University of Lublin, Nadbystrzycka 36, 20-618 Lublin, Poland e-mail: g.litak@pollub.pl

S. Schubert · G. Radons Institute of Physics, Chemnitz University of Technology, Reichenhainer Str. 70, 09126 Chemnitz, Germany

S. Schubert

e-mail:svs@physik.tu-chemnitz.de

\section{Introduction}

A cutting process is a basic machining technology to obtain the surface of the assumed parameters. In certain working conditions, it can be disturbed by chatter appearing as unexpected waves on the machined surface of a workpiece. The appearance of chatter was noticed and described by Taylor in the beginning of the twentieth century [1]. But the first approaches toward explanations of this phenomenon came about 50 years later through the analysis of self-sustained vibrations [2], regenerative effects [3], structural dynamics $[4,5]$, and finally the dry friction phenomenon $[6,7]$. Consequently, elimination and stabilization of the associated oscillations have become of high interest in science and technology [8-10]. The plausible adaptive control concept, based on relatively short time series [11], has been studied to gain deeper understanding.

Recently, apart from the widely developed chatter vibrations chaotic oscillations caused by various system nonlinearities were predicted and detected [12-20]. The recent technological demand is to improve the final surface properties of the workpiece and to minimize the production time with higher cutting speeds [21]. Thus, a better understanding of the physical phenomena associated with a cutting process becomes necessary [22]. In this paper, we will continue the work on chaotic instabilities in cutting processes proposing the $0-1$ test [23-27] as a tool identifying a possible chaotic solution [28]. 
This paper is organized as follows. After the present introduction (Sect. 1), we describe the model in Sect. 2. In Sect. 3, we provide the results of the simulations and corresponding power spectral densities (PSD) while in Sect. 4 the 0-1 test is applied, and subsequently the findings are confirmed by means of the multiscale entropy (Sect. 5). The paper ends with conclusions (Sect. 6).

\section{The model}

A regenerative cutting process may exhibit a wide range of complex behavior due to frictional effects [9, 12], structural nonlinearities [29], and delay dynamics [17, 18, 30-32]. Moreover, it may also involve loss of contact between the tool and the workpiece. The following equations model the regenerative cutting process and the mentioned properties.

After the first pass of the tool, the cutting depth can be expressed as

$h(t)=h_{0}-y(t)+y(t-\tau)$,

where $y(t-\tau)$ corresponds to the position of the workpiece during the previous pass, and $\tau$ is the time delay scaled by the period of revolution of the workpiece $2 \pi / \Omega_{0}$ (Fig. 1). The motion of the workpiece can be determined from the model proposed by Stépán [30]

$\ddot{y}+2 \gamma \dot{y}+\omega_{0}^{2} y=\frac{1}{m} \operatorname{sgn}\left(v_{0}-\dot{y}\right)\left(F_{y}(h)-F_{y}\left(h_{0}\right)\right)$,

$F_{y}(h)=\Theta(h) c_{1} w h^{3 / 4}$,

$\dot{y}\left(t^{+}\right)=-\beta \dot{y}\left(t^{-}\right)$,

where $\omega_{0}=\sqrt{k / m}$ is the frequency of free vibration, $v_{0}$ is the feed velocity, and $2 \gamma=c / m$ is the damping coefficient. $F_{y}(h)$ is the thrust force, which is the

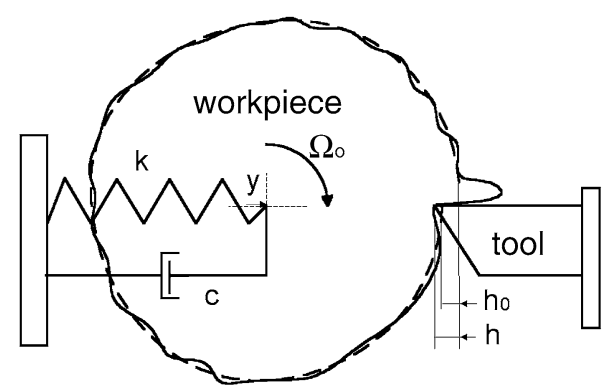

Fig. 1 Physical model of a regenerative cutting process [17] horizontal component of the cutting force, and $m$ is the effective mass of the workpiece. The thrust force $F_{y}$ is based on dry friction between the tool and the chip. It is assumed to have a power law dependence on the actual cutting depth $h$ and to be proportional to the chip width $w$ and a friction coefficient $c_{1} . \Theta(\cdot)$ denotes the Heaviside step function. The restitution parameter $\beta=0.75$ is associated with the impact after contact loss, while $t^{-}$and $t^{+}$denotes the time instants before and after the impact. Substituting (1) into (2), we derive a delay differential equation (DDE) for the workpiece motion $y(t)$. Plugging its solution into (1) results in the history of cutting depth $h(t)$.

\section{Simulation results}

The nonsmooth model equations are solved by a simple Euler integration scheme. The used parameters $[17,32]$ are presented in Table 1. Furthermore, the feed velocity $v_{0}$ has been assumed to be fairly large so that $v_{0}>\dot{y}$. Note that, in this case, the system nonlinearities are limited to the exponential dependence of the cutting force on the chip thickness and to the contact loss between the tool and workpiece.

The corresponding time series for two choices of the time delay parameter $\tau=1.8$ and $2.1 \mathrm{~ms}$ are presented in Fig. 2. These series have been plotted with points. On the first sight, one can notice that both solutions are complex but Fig. 2a shows points grouped in selected lines while the distribution of time history points of Fig. 2b looks more random. In Fig. $2 b, h$ reaches negative values that signal that the contact between the tool and the workpiece is lost. The power spectral densities (PSD) of cutting depth $S(\omega)=2 \pi / T|\mathcal{F}\{h(t)\}|^{2}$ for the two chosen delay times $(\tau=1.8 \mathrm{~ms} \text { and } \tau=2.1 \mathrm{~ms})^{1}$ indicate a

Table 1 Parameters used in the model

\begin{tabular}{ll}
\hline Parameter & Value \\
\hline initial cutting depth $h_{0}$ & $10^{-3} \mathrm{~m}$ \\
frequency of free vibration $\omega_{0}$ & $816 \mathrm{rad} / \mathrm{s}$ \\
damping coefficient $c$ & $86 \mathrm{~N} \mathrm{~s} / \mathrm{m}$ \\
effective mass of the workpiece $m$ & $17.2 \mathrm{~kg}$ \\
friction coefficient $c_{1}$ & $1.25 \times 10^{9} \mathrm{~N} / \mathrm{m}^{2}$ \\
chip width $w$ & $3.0 \times 10^{-3} \mathrm{~m}$ \\
\hline
\end{tabular}

${ }^{1} \mathcal{F}\{\cdot\}$ denotes the Fourier transform. 


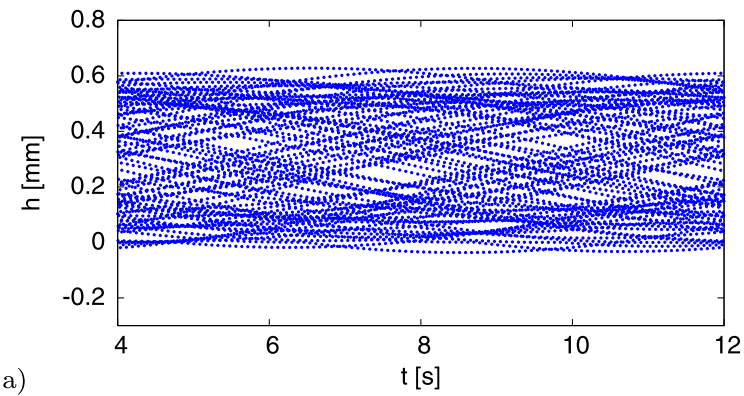

a)

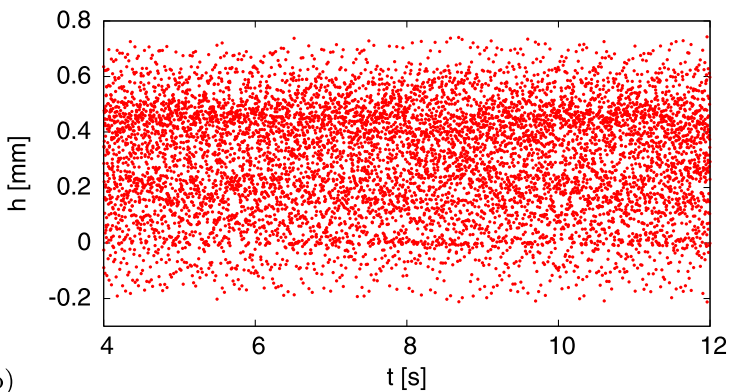

Fig. 2 Time series of cutting depth $h(t)$ for (a) time delay $\tau=1.8 \mathrm{~ms}$ and (b) $\tau=2.1 \mathrm{~ms}$ (sampling time $\Delta t=1 \mathrm{~ms}$ with an integration step $\Delta t / 10^{3}=1 \mu \mathrm{s}$ ) indicating regular and chaotic motion, respectively. The time series are plotted by sampling points

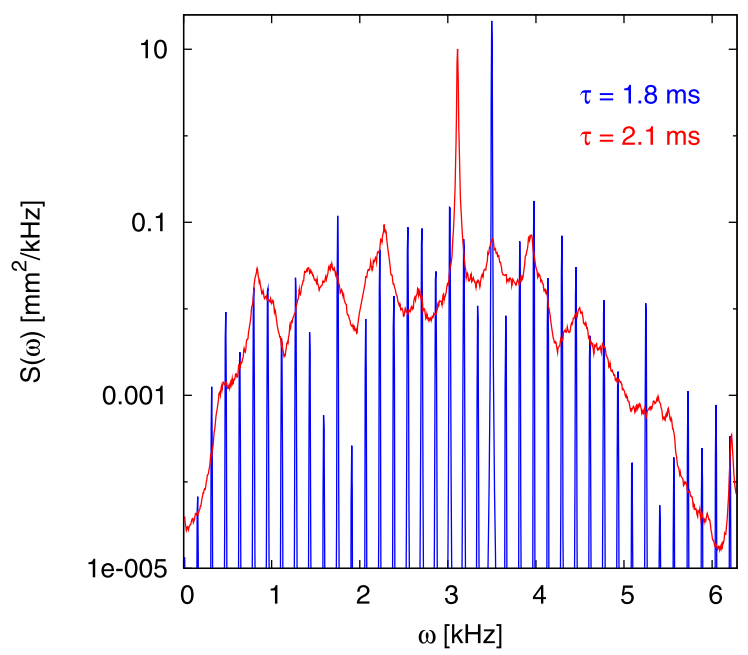

Fig. 3 Power spectral density $S(\omega)$ for two chosen delay times. A broad band spectral density indicates chaotic/stochastic dynamics whereas sharp peaks imply regular motion

transition from regular to chaotic motion. The sharp peaks in Fig. 3 belong to a high-periodic orbit (regular motion) whereas the broad spectrum indicates chaotic dynamics.
Both power spectra are dominated by a main peak. In case of regular motion, its position belongs to the delay time $\tau=1.8 \mathrm{~ms}$ while in case of chaotic dynamics the time scale belonging to the peak $\left(t_{p} \approx 2.0 \mathrm{~ms}\right)$ is smaller than the delay time $\tau=2.1 \mathrm{~ms}$. This smaller value could be a consequence of a tool-workpiece contact loss. Based on that, we take a closer look on other measures to characterize the model's dynamics and use a $0-1$ test for chaos to display a possible transition from regular to chaotic motion with increasing delay time $\tau$.

\section{Application of 0-1 test}

Based on the time series $\left\{\tilde{h}_{j}\right\}$ which is a discretization of the solution $h(t)$ of the DDE normalized by its standard deviation, we define dimensionless displacements in the $(p, q)$-plane in the following way [23, 24, 28]:

$p_{n}=\sum_{j=0}^{n} \tilde{h}_{j} \cos \left(j c_{0}\right), \quad q_{n}=\sum_{j=0}^{n} \tilde{h}_{j} \sin \left(j c_{0}\right)$,

where $c_{0}$ is a constant. In this way, regular dynamics is related to a bounded motion while any chaotic dynamics leads to an unbounded motion in the $(p, q)$-plane [23]; see Fig. 4a.

To obtain a quantitative description of the examined system, we perform calculations of the asymptotic properties defined by the total mean square displacement (MSD) $M(n)$, Fig. 4b, and finally we obtain the growth rate $K$ in the limit of large times

$$
\begin{gathered}
M(n)=\lim _{N \rightarrow \infty} \frac{1}{N} \sum_{j=1}^{N}\left[\left(p_{j+n}-p_{j}\right)^{2}\right. \\
\left.+\left(q_{j+n}-q_{j}\right)^{2}\right], \\
K=\lim _{n \rightarrow \infty} \frac{\ln (M(n)+1)}{\ln n} .
\end{gathered}
$$

For almost all values of the constant $c_{0}$, the parameter $K$ is approaching asymptotically 0 or 1 for regular or chaotic motion, respectively.

Note, practically, one has to truncate the sums in (4). Thus, we derived $K \approx 0.21$ for $\tau=1.8 \mathrm{~ms}$ and $K \approx 1.09$ for $\tau=2.1 \mathrm{~ms}$, which supports the first impression gained from the time series themselves, Fig. 2a and b. Note further that for delay time $\tau=$ $1.8 \mathrm{~ms}, K$ decays with increasing $n$ on much smaller 
values, Fig. 4c, which corroborates the result pointing toward regular motion.

Note that the parameter $c_{0}$ acts like a frequency in a spectral calculation, cp. (3). If it is badly chosen, $c_{0} / \Delta t$ resonates with one frequency of the process dynamics $\tilde{h}(t)$. Such a frequency belongs to a peak in the PSD, Fig. 3. In the 0-1 test, regular motion would yield a ballistic behavior in the $(p, q)$-plane and the corresponding quadratic growth of MSD results in an asymptotic growth rate. The disadvantage of the test, its strong dependence on the chosen parameter $c_{0}$, could be overcome by a proposed modification. Gottwald and Melbourne [24, 26] suggest to take several randomly chosen values of $c_{0}$ and compute the median of the belonging $K$-values. Particularly, in [26], the problems of averaging over $c_{0}$ as well as sampling the data points are discussed extensively. We followed this approach [26, 33], which improves the convergence of the test (Fig. 4c) without the consideration of longer time series, to find the time delay $\tau$ leading to chaos (see Fig. 5). We defined a modified square displacement $D(n)$ which exhibits the same asymptotic growth

$D\left(n, c_{0}\right)=M\left(n, c_{0}\right)-V_{\mathrm{osc}}\left(n, c_{0}\right)$,

where the oscillatory term $V_{\mathrm{osc}}\left(n, c_{0}\right)$ can be expressed by

$V_{\mathrm{osc}}\left(n, c_{0}\right)=E[\tilde{h}]^{2} \frac{1-\cos \left(n c_{0}\right)}{1-\cos \left(c_{0}\right)}$,

and $E[\tilde{h}]$ denotes the average of examined time series $\tilde{h}_{i}$

$E[\tilde{h}]=\frac{1}{N_{\max }} \sum_{i=1}^{N_{\max }} \tilde{h}_{i}$,

where $N_{\max }$ is the number of $\tilde{h}_{i}$ elements. Consequently, the oscillatory behavior is subtracted from the MSD $M\left(n, c_{0}\right)$ and the regression analysis of the linear growth of $D\left(n, c_{0}\right)$ (see (6)) with increasing $n$ is performed using the linear correlation coefficient which determines the value of $K_{c_{0}}$.

$K_{c_{0}}=\frac{\operatorname{cov}\left(\mathbf{X}, \mathbf{D}\left(c_{0}\right)\right)}{\sqrt{\operatorname{var}(\mathbf{X}) \operatorname{var}\left(\mathbf{D}\left(c_{0}\right)\right)}}$,

where vectors $\mathbf{X}=\left[1,2, \ldots, n_{\max }\right]$, and $\mathbf{D}\left(c_{0}\right)=$ $\left[D\left(1, c_{0}\right), D\left(2, c_{0}\right), \ldots, D\left(n_{\max }, c_{0}\right)\right]$.

The covariance $\operatorname{cov}(\mathbf{x}, \mathbf{y})$ and variance $\operatorname{var}(\mathbf{x})$, for arbitrary vectors $\mathbf{x}$ and $\mathbf{y}$ of $n_{\max }$ elements, and the corresponding averages $E[x]$ and $E[y]$, respectively, are defined
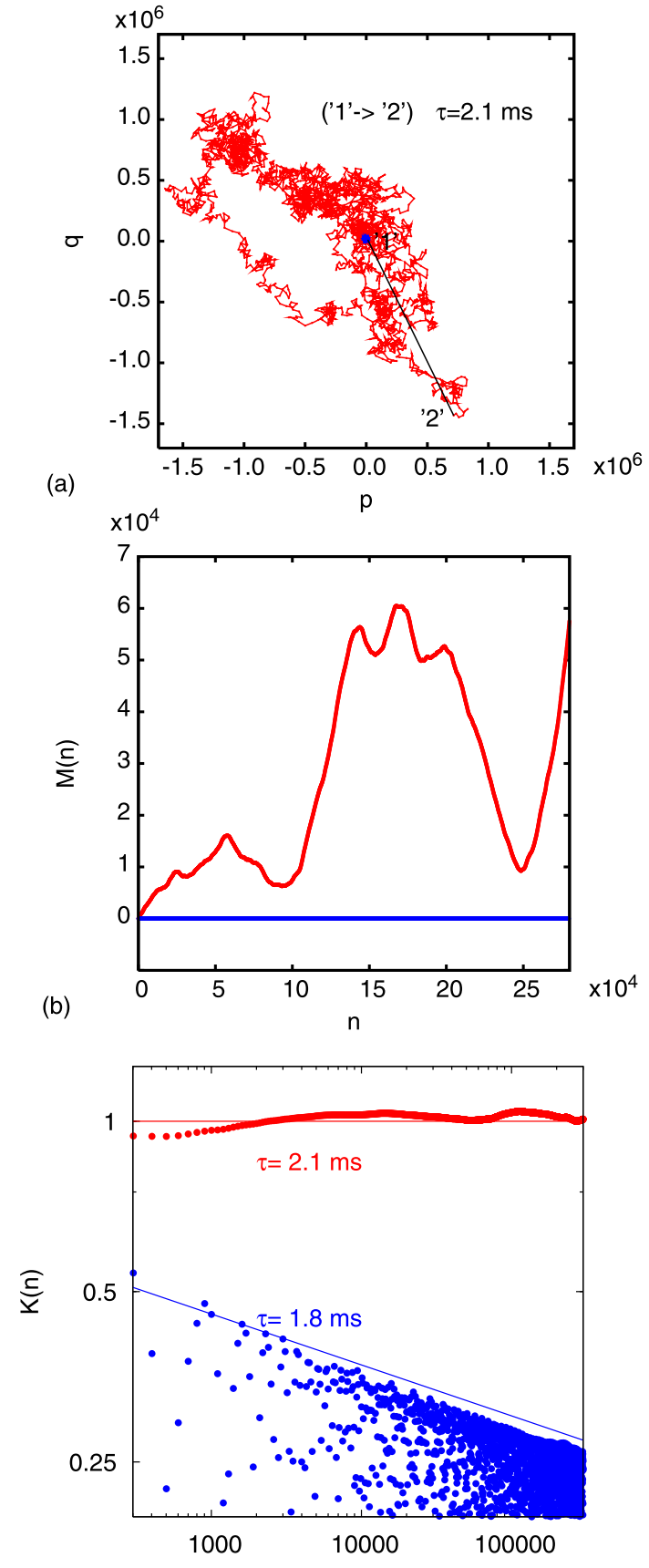

(c)

n

Fig. 4 (a) For regular motion ( $\tau=1.8 \mathrm{~ms}$ ) trajectories in $(p, q)$ stay bounded around the initial point " 1 ." For chaotic time series ( $\tau=2.1 \mathrm{~ms}$ ), trajectories in $p$ and $q$ coordinates show Brownian motion-like behavior. (b) Thus, the mean square displacement $M(n)$ increases with time. For estimation of $p_{n}$ and $q_{n}$, (3), we used $c_{0}=0.7$ and for $M(n)$ and $K,(4)$ and (5), the upper limits of $N, n$ are $N_{\max }=40000, n_{\max }=280000$, respectively. (c) For regular dynamics ( $\tau=1.8 \mathrm{~ms}$ ), the $K$-value converges toward zero with increasing time. For chaotic dynamics $(\tau=2.1 \mathrm{~ms})$, $K$ stays close to 1 


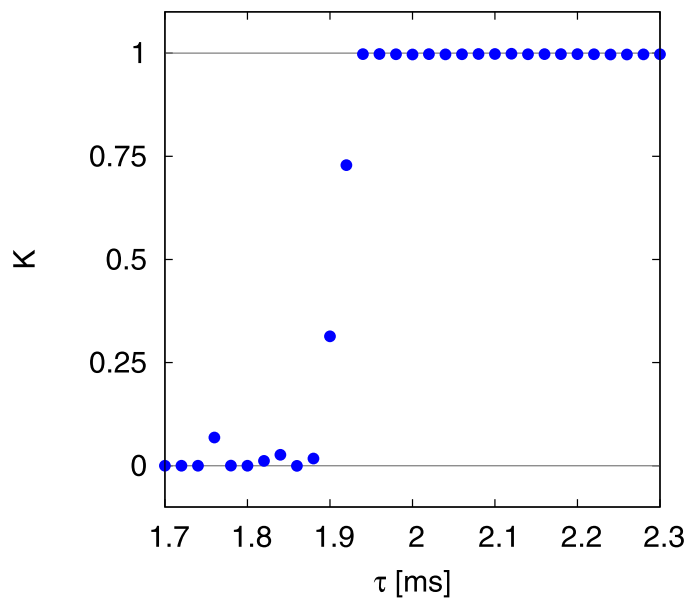

Fig. $5 K$-values for different delay times $\tau$ indicate a transition from regular to chaotic dynamics in the region of $1.9 \mathrm{~ms}$

$\operatorname{cov}(\mathbf{x}, \mathbf{y})=\frac{1}{n_{\max }} \sum_{n=1}^{n_{\max }}(x(n)-E[x])(y(n)-E[y])$,

$\operatorname{var}(\mathbf{x})=\operatorname{cov}(\mathbf{x}, \mathbf{x})$.

Finally, the median is taken of $K_{c_{0}}$-values (see (9)) corresponding to 100 different values of $c_{0} \in(0, \pi)$. The results of $K$ for different delay times $\tau$, Fig. 5, in the window between $1.75 \mathrm{~ms}$ and $2.3 \mathrm{~ms}$ indicates a transition from regular to chaotic dynamics with increasing delay time in the region of $1.9 \mathrm{~ms}$.

As a consequence, we conclude that in the investigated window increasing cutting speed leads to a transition from chaotic chatter dynamics to regular motion with improved surface quality.

\section{Multiscale entropy}

To characterize the solutions of the DDE, (1) and (2), with regard to information production rate and complexity, we aim to calculate multiscale entropy (MSE) [34]. This method was successfully applied to analyze the complexity of biological signals [34, 35]. It is suitable for short and noisy time series. As a consequence, the chosen procedure would be applicable to experimental data as well. We use an algorithm provided by PhysioNet [36]. First, we compute coarsegrained time series $\left\{x^{(N)}\right\}$ using nonoverlapping intervals containing $N$ equidistant data points $h_{i}$,
$x_{j}^{(N)}=\frac{1}{N} \sum_{i=(j-1) N+1}^{j N} h_{i}$.

In the next step, we calculate sample entropy $S_{E}^{(N)}$ [37] for these coarse-grained time series. Sample entropy is the negative of the logarithm of the conditional probability that sequences of $m$ consecutive data points $\mathbf{x}_{i}^{(N)}=\left(x_{i}^{(N)}, \ldots, x_{i+m-1}^{(N)}\right)$ and $\mathbf{x}_{j}^{(N)}$ close to each other will also be close to each other when one more point is added to them. Hence, it is estimated as follows:

$S_{E}^{(N)}(m, r)=-\ln \frac{U_{m+1}^{(N)}(r)}{U_{m}^{(N)}(r)}$,

where $U_{m}^{(N)}(r)$ represents the relative frequency that a vector $\mathbf{x}_{i}^{(N)}$ is close to a vector $\mathbf{x}_{j}^{(N)}(i \neq j)$. Close to each other in the sense that their infinity norm distance is less than $\varepsilon=r \sigma$. By $\sigma$, we denote the standard deviation of the data. In the limit of $m \rightarrow \infty$ and $r \rightarrow 0$, sample entropy is equivalent to order-2 Rényi entropy $K_{2}$ and is suitable to characterize the system's dynamics [38]. For independent variables $\{\xi\}$, the entropy follows from $S_{E}^{(N)}(m, r)=-\ln P\left(\mid \xi_{i}^{(N)}-\right.$ $\xi_{j}^{(N)} \mid<\varepsilon$ ) and is independent of word length $m$. For Gaussian white noise (GWN), the coarse-grained time series is known to be Gaussian distributed, also. For small $\varepsilon$, this yields $S_{E}^{(N)}(m, r) \approx-\ln \left[\varepsilon /\left(\sqrt{\pi} \sigma^{(N)}\right)\right]$. Using that the standard deviation of the coarse-grained time series $\sigma^{(N)}$ decreases with $1 / \sqrt{N}$ leads to following expression:

$S_{E}^{(N)}(m, r) \approx-\ln \left(r \sqrt{\frac{N}{\pi}}\right) \quad(r \rightarrow 0)$.

To clear up the characteristics of the cutting process, we look at MSE depending on box size $r$ for the two chosen delay times, Fig. 6. For regular motion, we expect the entropy to approach zero with decreasing $r$. This is observed for the time series with delay time $\tau=1.8 \mathrm{~ms}$. For chaotic dynamics, the entropy should stay finite, observed for $\tau=2.1 \mathrm{~ms}$. For the sake of completeness, it should be mentioned that in the case of stochastic dynamics the entropy would diverge with decreasing spatial resolution $r$, cp. (13). In Figs. 6 and 7, we further analyze the scale factor dependence of MSE. The entropic measure is always larger for the chaotic time series since it is the more complex one. MSE for small scale factor, Fig. 7a, indicates that there is no characteristic time scale, comparable to $1 / f$-noise [34]. But for larger scale factors, 


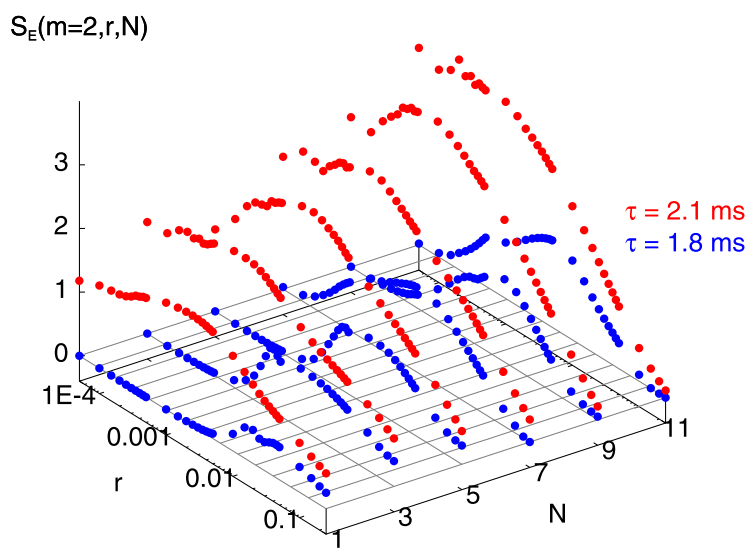

Fig. 6 Multiscale entropy $S_{E}$ depending on scale factor $N$ and box size $r$. For regular motion, we expect the entropy to approach zero with decreasing $r$. For chaotic dynamics, the entropy should stay finite. Since $S_{E}$ is not decreasing with scale factor significantly, it seems there is no characteristic time scale present in the data

MSE is decaying comparable to Gaussian white noise, Fig. 7b. Thus, even in the chaotic case, there exists a characteristic time scale which is close to the delay time. The frequencies dominating $S(\omega)$, Fig. 3, are also present in $S_{E}^{(N)}(m, r)$. They belong to minima in Fig. 7. We learn coarse-graining of the data over multiple of time scale $t_{p}$ belonging to structures in the cutting process dynamics leads to less complex time series and contains less information.

\section{Conclusions and last remarks}

Concluding, the 0-1 test differentiates between the two types of motion. Depending on the chosen delay time for the investigated DDE, (1) and (2), regular or chaotic motion is observed and a transition from chaotic to regular motion is detected with increasing cutting speed. The nature of solutions has been also confirmed by the corresponding power spectral densities and multiscale entropies. The latter reveals more insights into the process dynamics but is of much higher computational cost than the $0-1$ test and the spectral calculations.

The $0-1$ test appeared to be relatively simple, and consequently, useful for systems with delay and discontinuities. A huge advantage of the test is its low computational effort and the possibility to compute it "on the fly" while the data is still growing. One of the

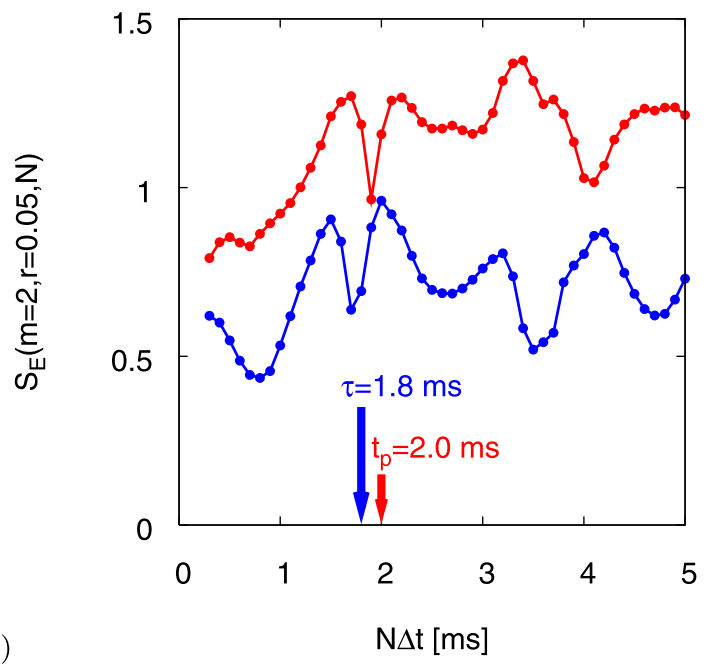

(a)

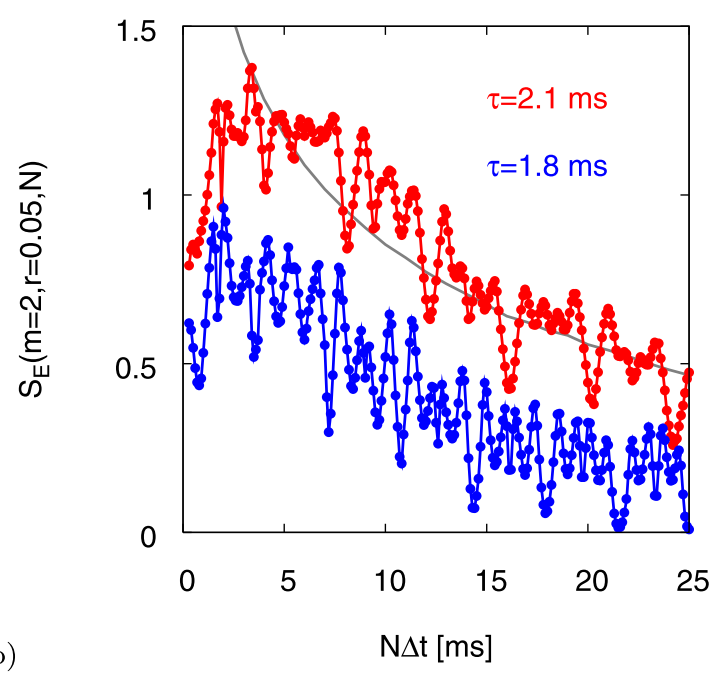

Fig. 7 Multiscale entropy $S_{E}$ for fixed $m=2$ and $r=0.05$ depending on scale factor $N$. The time series with delay time $\tau=2.1 \mathrm{~ms}$ seems to be more complex than the time series with delay time $\tau=1.8 \mathrm{~ms}$ since its entropy is larger. (a) To gain a higher scale factor resolution, we recorded also time series with smaller sampling time $\Delta t=0.1 \mathrm{~ms}$. (b) The existence of a characteristic time scale which is in order of magnitude of the delay time is indicated by the decay of entropy with increasing scale factor $N$. The gray line represents GWN with $r=0.25$

useful aspects of the $0-1$ test is that the result can be plotted against the parameter $\tau$.

The presented method gives a quantitative criterion for chaos similar to the maximum Lyapunov exponent.

As demonstrated by Falconer et al. [25] and Krese and Govekar [33], the method can be used on experimental data as well. Unfortunately, in case of the cutting process, experimental data are often characterized 
by a relatively high level of noise [20]. In the examined system, we waived the possibility of additive noise. It was shown that the $0-1$ test could be applied on dynamical systems with additive noise and a good signal to noise ratio [24].

Acknowledgements This work is partially supported by the European Union within the framework of the Integrated Regional Development Operational Program as project POIG.0101. $02-00-015 / 08$ and by the 7th Framework Programme FP7REGPOT-2009-1, under Grant Agreement No. 245479.

Open Access This article is distributed under the terms of the Creative Commons Attribution License which permits any use, distribution, and reproduction in any medium, provided the original author(s) and the source are credited.

\section{References}

1. Taylor, F.: On the art of cutting metals. Trans. Am. Soc. Mech. Eng. 28, 31-350 (1907)

2. Arnold, R.N.: The mechanism of tool vibration in the cutting of steel. Proc. Inst. Mech. Eng. 154, 261-284 (1946)

3. Tobias, S.A., Fishwick, W.: A Theory of Regenerative Chatter. The Engineer, London (1958)

4. Tlusty, J., Polacek, M.: The stability of machine tool against self-excited vibrations in machining. ASME Int. Res. Prod. Eng. 465-474 (1963)

5. Merrit, H.E.: Theory of self-excited machine-tool chatter. ASME J. Eng. Ind. 87, 447-454 (1965)

6. Wu, D.W., Liu, C.R.: An analytical model of cutting dynamics. Part 1: Model building. ASME J. Eng. Ind. 107, 107-111 (1985)

7. Wu, D.W., Liu, C.R.: An analytical model of cutting dynamics. Part 2: Verification. ASME J. Eng. Ind. 107, 112118 (1985)

8. Altintas, Y.: Manufacturing Automation: Metal Cutting Mechanics, Machine Tool Vibrations, and CNC Design. Cambridge University Press, Cambridge (2000)

9. Warminski, J., Litak, G., Cartmell, M.P., Khanin, R., Wiercigroch, W.: Approximate analytical solutions for primary chatter in the nonlinear metal cutting model. J. Sound Vib. 259, 917-933 (2003)

10. Insperger, T., Gradisek, J., Kalveram, M., Stépán, G., Winert, K., Govekar, E.: Machine tool chatter and surface location error in milling processes. J. Manuf. Sci. Eng. 128, 913-920 (2006)

11. Ganguli, A., Deraemaeker, A., Preumont, A.: Regenerative chatter reduction by active damping control. J. Sound Vib. 300, 847-862 (2007)

12. Grabec, I.: Chaotic dynamics of the cutting process. Int. J. Mach. Tools Manuf. 28, 19-32 (1988)

13. Tansel, I.N., Erkal, C., Keramidas, T.: The chaotic characteristics of three-dimensional cutting. Int. J. Mach. Tools Manuf. 32, 811-827 (1992)

14. Gradisek, J., Govekar, E., Grabec, I.: Time series analysis in metal cutting: chatter versus chatter-free cutting. Mech. Syst. Signal Process. 12, 839-854 (1998)
15. Gradisek, J., Govekar, E., Grabec, I.: Using coarse-grained entropy rate to detect chatter in cutting. J. Sound Vib. 214, 941-952 (1998)

16. Marghitu, D.B., Ciocirlan, B.O., Craciunoiu, N.: Dynamics in orthogonal turning process. Chaos Solitons Fractals 12, 2343-2352 (2001)

17. Litak, G.: Chaotic vibrations in a regenerative cutting process. Chaos Solitons Fractals 13, 1531-1535 (2002)

18. Fofana, M.S.: Delay dynamical systems and applications to nonlinear machine-tool chatter. Chaos Solitons Fractals 12, 731-747 (2003)

19. Gradisek, J., Grabec, I., Sigert, I., Friedrich, R.: Stochastic dynamics of metal cutting: bifurcation phenomena in turning. Mech. Syst. Signal Process. 16, 831-840 (2002)

20. Litak, G., Rusinek, R., Teter, A.: Nonlinear analysis of experimental time series of a straight turning process. Meccanica 39, 105-112 (2004)

21. Stépán, G., Szalai, R., Insperger, T.: Nonlinear dynamics of high-speed milling subjected to regenerative effect. In: Radons, G. (ed.) Nonlinear Dynamics of Production Systems. Wiley, New York (2003)

22. Vela-Martínez, L., Jáuregui-Correa, J.C., GonzálezBrambila, O.M., Herrera-Ruiz, G., Lozano-Guzmán, A.: Instability conditions due to structural nonlinearities in regenerative chatter. Nonlinear Dyn. 56, 415-427 (2009)

23. Gottwald, G.A., Melbourne, I.: A new test for chaos in deterministic systems. Proc. R. Soc. Lond. A 460, 603-611 (2004)

24. Gottwald, G.A., Melbourne, I.: Testing for chaos in deterministic systems with noise. Physica D 212, 100-110 (2005)

25. Falconer, I., Gottwald, G.A., Melbourne, I., Wormnes, K.: Application of the 0-1 test for chaos to experimental data. SIAM J. Appl. Dyn. Syst. 6, 95-402 (2007)

26. Gottwald, G.A., Melbourne, I.: On the implementation of the 0-1 test for chaos. SIAM J. Appl. Dyn. Syst. 8, 129-145 (2009)

27. Gottwald, G.A., Melbourne, I.: On the validity of the 01 test for chaos. Nonlinearity 22, 1367-1382 (2009)

28. Litak, G., Syta, A., Wiercigroch, M.: Identification of chaos in a cutting process by the $0-1$ test. Chaos Solitons Fractals 40, 2095-2101 (2009)

29. Pratt, J.R., Nayfeh, A.H.: Chatter control and stability analysis of a cantilever boring bar under regenerative cutting conditions. Philos. Trans. R. Soc. Lond. A 359, 759-792 (2001)

30. Stépán, G.: Modelling nonlinear regenerative effects in metal cutting. Philos. Trans. R. Soc. Lond. A 359, 739-757 (2001)

31. Wang, X.S., Hu, J., Gao, J.B.: Nonlinear dynamics of regenerative cutting processes-Comparison of two models. Chaos Solitons Fractals 29, 1219-1228 (2006)

32. Litak, G., Sen, A.K., Syta, A.: Intermittent and chaotic vibrations in a regenerative cutting process. Chaos Solitons Fractals 41, 2115-2122 (2009)

33. Krese, B., Govekar, E.: Nonlinear analysis of laser droplet generation by means of $0-1$ test for chaos. Nonlinear Dyn. 67, 2101-2109 (2012)

34. Costa, M., Goldberger, A.L., Peng, C.-K.: Multiscale analysis of complex biological signals. Phys. Rev. Lett. 89, 068102 (2002) 
35. Costa, M., Goldberger, A.L., Peng, C.-K.: Multiscale analysis of biological signals. Phys. Rev. E 89, 021906 (2005)

36. Goldberger, A.L., Amaral, L.A.N., Glass, L., Hausdorff, J.M., Ivanov, P.Ch., Mark, R.G., Mietus, J.E., Moody, G.B., Peng, C.-K., Stanley, H.E.: PhysioBank, physioToolkit, and physioNet: Components of a new research resource for complex physiologic signals. Circulation 101, 215-220 (2000)
37. Richman, J.S., Moorman, J.R.: Physiological time-series analysis using approximate entropy and sample entropy. Am. J. Physiol. 278, H2039-H2049 (2000)

38. Grassberger, P., Procaccia, I.: Estimation of the Kolmogorov-entropy from a chaotic signal. Phys. Rev. A 28, 2591-2593 (1983) 